\title{
Revisión
}

\section{Otitis media: conceptos actuales}

Hernán Sierra- Fernández ${ }^{1}$, Malka Schultz- Faingezicht ${ }^{2}$, C ecilia Loaiza- Mendoza ${ }^{3}$, Adriano Arguedas- Mohs., ${ }^{4}$

\section{R e s u m e n}

1. Médico General, Instituto de Atención Pediátrica

2. Farmacéutica, Instituto de Atención Pediátrica

3. Médico Pediatra, Instituto de Atención Pediátrica

4. Pediatra Infectólogo, Instituto de Atención Pediátrica

5. Profesor, Universidad de Ciencias Médicas

Abreviaturas: $0 \mathrm{MA}$, otitis media aguda; 0 MR,otitis media recurrente; $\mathrm{OMF}$, otitis media falla terapéutica.

Correspondencia: Adriano Arguedas Mohs. Pediatra Infectólogo, Director Instituto de Atención Pediátrica. A partado Postal \#6071150 La Uruca, San José, Costa Rica.Fax (506)221-3894. E-mail: aarguedas@ iped.net

ISSN 0001-6002/2004/46/3/125-131 Acta Médica Costarricense,@2004 Colegio de Médicos y Cirujanos
La otitis media aguda es uno de los motivos más frecuentes de consulta en la edad pediátrica. Los gérmenes que con mayor frecuencia se aíslan en el oído medio de estos pacientes son el Streptococcus pneumoniae, el Haemophilus influenzae, la Moraxella catarrhalis y el Streptococcus pyogenes. El patrón de resistencia antibiótica de estas bacterias varía dependiendo de la región geográfica, y es la base fundamental para establecer recomendaciones terapéuticas. El análisis en Costa Rica, de la microbiología de la otitis media aguda, otitis media recurrente y otitis media catalogada como falla terapéutica, sugiere que la amoxicilina en una dosis de $50 \mathrm{mg} / \mathrm{Kg} /$ día por 10 días, debe ser considerada el antibiótico de primera línea en los casos de otitis media aguda. En los pacientes con otitis media recurrente, falla antimicrobiana o cuando se sospeche la presencia de un Streptococcus pneumoniae resistente a la penicilina, se debe considerar el uso de amoxicilina en dosis más elevadas, amoxicilina con ácido clavulánico, macrólidos o ceftriaxona.

Descriptores: otitis media aguda, otitis media recurrente, otitis media falla terapéutica.

Recibido: 10 de febrero de 2004

Aceptado: 27 de abril de 2004

El término otitis media aguda se refiere a la inflamación del oído medio asociada a signos y síntomas locales o sistémicos, secundaria a un proceso infeccioso en el oído medio producido por agentes virales o bacterianos. OMA es la entidad infecciosa más frecuente en la edad pediátrica ${ }^{1,2}$. Solo en los Estados Unidos de América ocurren más de 30 millones de consultas por otitis media al año, y se estima que esta patología representa el 18\% de las consultas médicas en la edad preescolar ${ }^{3,45}$. En Finlandia, cuya población es de aproximadamente 5 millones, el impacto económico de la otitis media aguda se estima en 138 millones de dólares por año, cifra que en Estados Unidos, supera los 2 billones de dólares ${ }^{3,6,7}$.Además del impacto económico, la otitis media aguda puede producir complicaciones tan severas como meningitis, mastoiditis, trombosis del seno lateral, absceso cerebral, empiema subdural, pérdida de la audición y trastornos del habla ${ }^{1}$.

Por su frecuencia, tal patología está sujeta constantemente a una gran cantidad de procesos de investigación, lo que ha generado diferentes criterios con respecto al diagnóstico, microbiología y manejo de la OMA, OMR y OMF. La OMR se define como la historia de tres episodios de otitis media aguda en seis meses o cuatro episodios en un año, y la OMF como un episodio de otitis media aguda que no mejora luego de 72 horas de tratamiento con antibióticos, o que recae en un período no mayor a 14 días, después de la última toma de antibiótico para el tratamiento de un episodio previo.

Esta revisión tiene como finalidad analizar aspectos generales de la enfermedad, estableciendo el comportamiento bacteriológico en niños a nivel mundial y específicamente en Costa Rica, para, con base en estos datos, emitir recomendaciones acerca del adecuado manejo antimicrobiano y preventivo de los pacientes. 


\section{Fisiopatología}

El oído medio es una cavidad entre el conducto auditivo externo y el oído interno. En condiciones basales, esta cavidad es estéril y mantiene presiones normales para la óptima conducción del sonido. Para esto, la trompa de eustaquio se desempeña como reguladora de la presión, al comunicar el oído medio con la nasofaringe, manteniendo así una adecuada ventilación. Este conducto actúa también como drenaje de secreciones del oído medio y su mucosa evita la llegada de agentes patógenos por la función de barrido ${ }^{8}$. Las funciones normales del tubo se pueden alterar por una mala posición, por cambios de presión o por lesiones de su mucosa provocadas por factores externos, como la nicotina o las infecciones virales. Cuando el funcionamiento normal de la trompa de eustaquio se ve afectado por alguno de los mecanismos descritos, agentes bacterianos que residen habitualmente en la nasofaringe, pueden llegar hasta el oído medio induciendo un proceso inflamatorio mediado por las interleukinas 1, 6, 8 y factor de necrosis tumoral alfa ${ }^{9,10}$. Este proceso se acentúa en presencia de proteínas bacterianas, como la autolisina y la pneumolisina del Streptococcus pneumoniae ${ }^{11,12}$, o en el caso de infecciones por Haemophilus influenzae, en las que hay un aumento en la cantidad de receptores de interleukina 8, producido por la pared celular bacteriana ${ }^{13}$.

La mayoría de las OMAson precedidas por una infección viral de las vías respiratorias superiores y el pico máximo de incidencia es en niños menores de 3 años ${ }^{14,15,16,17}$, debido a que en ellos la trompa de eustaquio está en posición más horizontal que en el adulto, lo que no permite un óptimo drenaje. Además, la falta de rigidez del cartílago en el oído medio de los niños dificulta la apertura del tubo al deglutir e impide una adecuada ventilación ${ }^{8}$. Otro factor es la inmadurez inmunológica propia de esta edad, que permite una mayor colonización de la nasofaringe ${ }^{18}$. Varios estudios han demostrado que la colonización nasofaríngea con organismos resistentes, aumenta en niños que asisten a guarderías o que tienen hermanos que lo hacen, por lo que se puede considerar un factor de riesgo en este grupo de niños con otitis ${ }^{18,19}$. Otros factores de riesgo son la predisposición familiar y el uso de la chupeta, que genera una presión negativa durante la succión ${ }^{20}$.

\section{Microbiología}

Debido a que la frecuencia y patrones de resistencia de las bacterias que producen otitis media varían en diferentes partes del mundo, es fundamental conocer el patrón microbiológico en cada región geográfica. Los agentes bacterianos que se aíslan con mayor frecuencia del oído medio de pacientes con otitis son Streptococcus pneumoniae, Haemophilus influenzae no tipificable, Moraxella catarrhalis y el Streptococcus pyogenes. A pesar de existir variaciones regionales ${ }^{21} \mathrm{y}$ por patología (OMA, OMR u OMF), se sabe que generalmente el $S$. pneumoniae y el $H$. influenzae predominan sobre los otros dos gérmenes (Figura 1).
En Costa Rica, desde 1992 se han venido recopilando datos sobre la microbiología del oído medio en niños costarricenses con OMA, OMR y OMF ${ }^{22,23}$. En pacientes con OMA u OMR el germen más frecuente es el Streptococcus pneumoniae, seguido del Haemophilus influenzae, mientras que en pacientes con OMF el Haemophilus influenzae es el más frecuente. La Moraxella catarrhalis es infrecuente, siendo superada por el Streptococcus pyogenes (Figura 2).

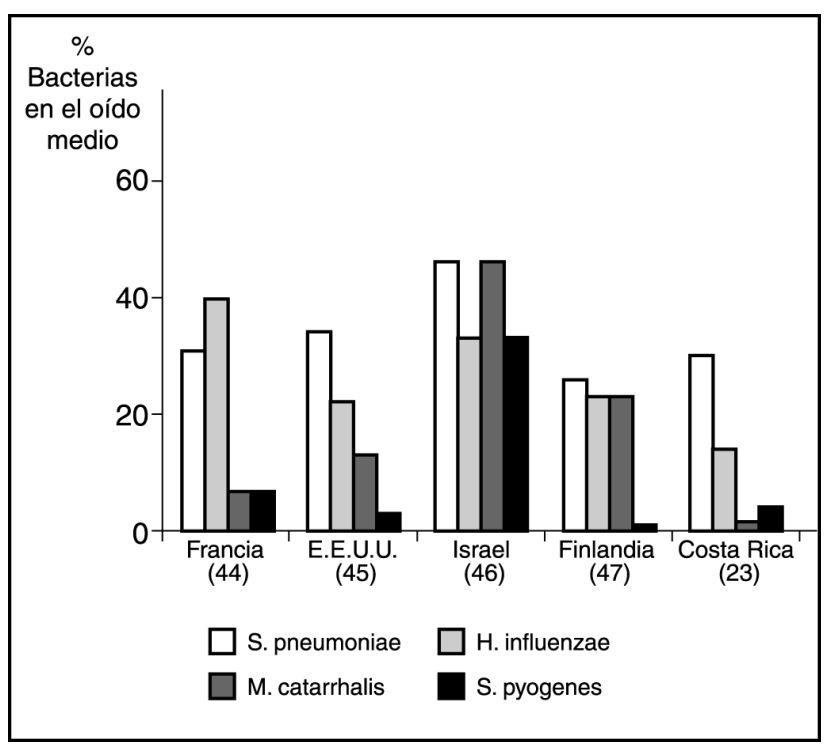

Figura 1: Frecuencia de bacterias aisladas en el oído medio de niños con otitis media.

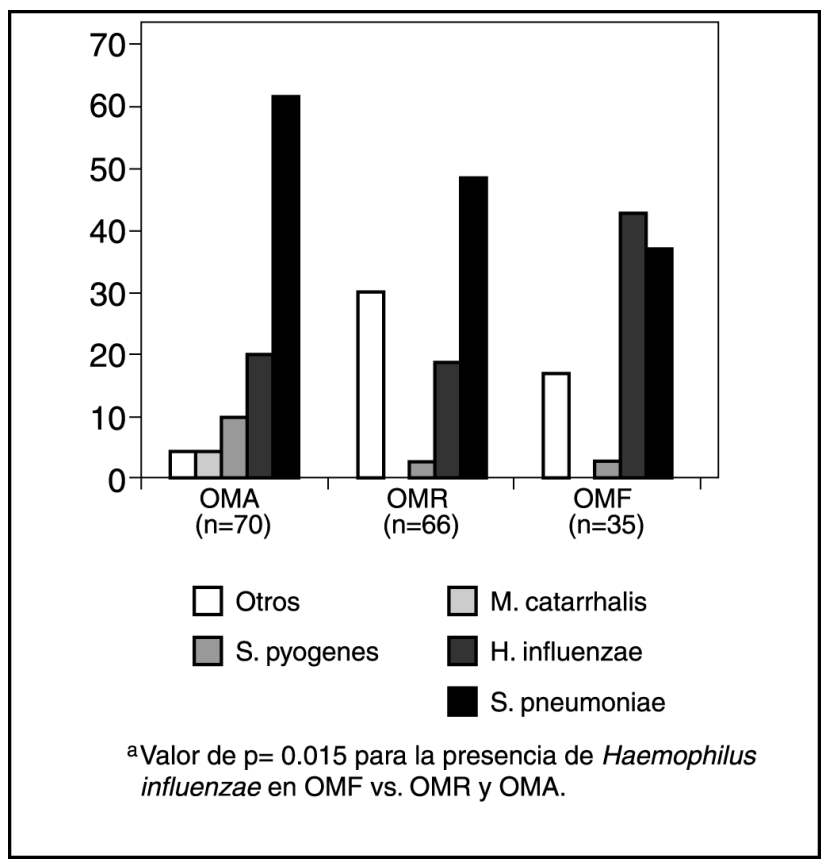

Figura 2: Microbiología del oído medio en niños costarricenses con otitis media aguda (OMA), otitis media recurrente (OMR) y falla terapéutica (OMF). ${ }^{\text {a }}$ 


\section{Serología de Streptococcus pneumoniae}

Recientemente la FDA aprobó el uso de una nueva vacuna conjugada contra $S$. pneumoniae, en niños a partir de los dos meses de edad. Debido a que esta vacuna cubre únicamente siete serotipos de $S$. pneumoniae, es importante conocer cuáles son los serotipos más frecuentes en diversas enfermedades y regiones del mundo. En el caso específico de la OMA, los serotipos 19F y 23F son los más recurrentes a nivel mundial. Datos recolectados en niños costarricenses con OMA, OMR u OMF durante el período 1999-2001 ${ }^{23}$, reflejaron que en esa época el serotipo 19F representó aproximadamente el $85 \%$ del total de cepas aisladas. Información aún no publicada de cepas de $S$. pneumoniae aisladas del líquido de oído medio durante 2002 y 2003, evidencia una reducción muy significativa del serotipo $19 \mathrm{~F}$ a un $27 \%$. Durante ese mismo periodo, otros serotipos de $S$. pneumoniae detectados frecuentemente fueron el $6 \mathrm{~B}(15 \%)$, el $9 \mathrm{~V}(9.5 \%)$ y el $16 \mathrm{~F}$ $(9.5 \%)$.

\section{Patrones de resistencia}

Los porcentajes de sensibilidad de gérmenes aislados del oído medio son la base fundamental para establecer recomendaciones terapéuticas en cada región del mundo. En los últimos años ha existido preocupación por el incremento de cepas resistentes a antibióticos encontrados en la nasofaringe y en el oído medio de ciertos grupos de alto riesgo.

En un inicio, en la década de los ochenta, el problema principal fueron las cepas de $H$. influenzae y $M$. catarrhalis productoras de beta lactamasa y más recientemente, cepas de S. pneumoniae resistentes a la penicilina. En los Estados Unidos el porcentaje de cepas de $\mathrm{H}$. influenzae productoras de beta lactamasa es de un $47 \%$, mientras que los porcentajes a nivel mundial varían desde un $13 \%$ en Europa Central, hasta un $26 \%$ en Israel ${ }^{22}$ y un $12 \%$ en Chile ${ }^{24}$. En Costa Rica el $H$. influenzae ha tenido un comportamiento peculiar y claramente definido. Durante el periodo comprendido entre 1992 y $1997^{22}$, las cepas de $H$. influenzae tipo B representaban un 34\% del total de cepas de H. influenzae detectadas. En 1998 la vacuna conjugada fue introducida al esquema nacional de vacunación y para el segundo segmento del análisis (1999$2001)^{23}$ este porcentaje de $H$. influenzae tipo B bajó a un $0 \%$. El segundo punto importante con esta bacteria es que el porcentaje de cepas productoras de beta lactamasa en Costa Rica, se ha mantenido bajo (7\%) durante estos nueve años ${ }^{22,23}$.

En el caso de S. pneumoniae, en los últimos años se han presentado múltiples estudios que demuestran un aumento en el número de cepas resistentes a penicilina y otros antibióticos ${ }^{24}$, particularmente en ciertos pacientes tales como: a) niños menores de dos años, b) que asisten a guarderías y c) con historia de haber recibido tratamiento con algún antibiótico en los últimos tres meses ${ }^{25}$. Es importante enfatizar que en el caso de $S$. pneumoniae existen tres tipos de patrones de susceptibilidad a penicilina: a) Los susceptibles a penicilina
(Concentración Inhibitoria Mínima [CIM]: < 0,06 mg/L), b) los de resistencia intermedia a penicilina (CIM: $0.125-\leq 1$ $\mathrm{mg} / \mathrm{L}$ ) y c) los resistentes (CIM: >1 mg/L). Alrededor del mundo los patrones de resistencia del Streptococcus pneumoniae a la penicilina, varían de un $31 \%$ en Europa Central a un $20-40 \%$ en Estados Unidos y a un $52 \%$ en Israel ${ }^{21}$. En Costa Rica hemos documentado un incremento importante de resistencia a la penicilina. En el período 1992-1997 solo el 19\% de las cepas de $S$. pneumoniae aisladas del oído medio eran resistentes a penicilina, con cerca de un 2\% de cepas altamente resistentes. Datos analizados en el período 1999-2001 indican que en pacientes con otitis media aguda, el $42 \%$ de las cepas eran resistentes a la penicilina, mientras que en los pacientes con otitis media recurrente y otitis media con falla terapéutica, el porcentaje de resistencia a la penicilina fue del $63 \%$ y el 64\%, respectivamente (Figura 3). Además de lo anterior, detectamos un aumento importante en el porcentaje de resistencia a trimetoprim-sulfametoxazol, sin modificarse el porcentaje de resistencia a macrólidos, cefalosporinas o betalactámicos ${ }^{23}$. En el caso de las cepas de Streptococcus pneumoniae resistentes a la penicilina, los porcentajes de resistencia a otros antibióticos utilizados en el tratamiento de estos pacientes fue de un $0 \%$ a la amoxicilina, ceftriaxona y gatifloxacina; de un $4.5 \%$ a los macrólidos, y de un $8.5 \%$ al cefuroxime ${ }^{23}$.

\section{Diagnóstico}

El dilema de la otitis media es que, a pesar de sus diferentes etiologías, la clínica es muy similar en todos los casos y presenta solo pocas diferencias. Muchos autores han descrito la clínica de esta enfermedad con base en síntomas y valoración de la membrana timpánica. De este modo, se habla de la presencia de fiebre, dolor, tirar del oído, irritabilidad, cambios en el apetito, cambios en el sueño, tímpano eritematoso, presencia de exudado y opacidad del tímpano, sin embargo, ninguna de estas es específica de la etiología bacteriana o viral ${ }^{26}$.

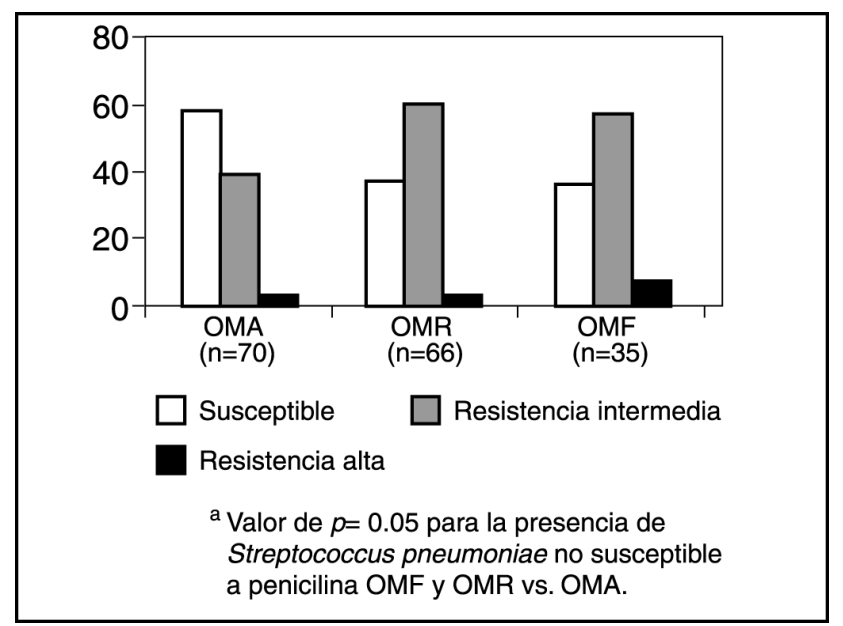

Figura 3: Susceptibilidad a penicilina de Streptococcus pneumoniae aislado del oído medio en niños costarricenses con otitis media aguda $(\mathrm{OMA})$, otitis media recurrente (OMR) y falla terapéutica (OMF). a 
Como consecuencia y aunado a otras razones como el temor a complicaciones, la ansiedad de la familia ante la situación y la falta de un consenso de criterios diagnósticos, algunas veces se da tratamiento antimicrobiano en forma innecesaria. De hecho, se calcula que de los pacientes con otitis media aguda tratados con antibióticos, el $25 \%$ no requiere tratamiento antimicrobiano ${ }^{1}$.

Debido a lo anterior, es importante recordar que para hablar de otitis media aguda, se debe confirmar que hay inflamación del oído medio y presencia de líquido, además de signos y síntomas locales o sistémicos ${ }^{27,28,29}$. Para diagnosticar una otitis media aguda de origen bacteriano, se requiere la presencia de abombamiento del tímpano u otorrea purulenta ${ }^{30}$, más cualquiera de los signos mencionados. Se menciona que el abombamiento de la membrana timpánica es el hallazgo que más se asocia con una etiología bacteriana ${ }^{26}$.

La ausencia de estos signos debe hacer pensar en una etiología viral o en un diagnóstico equivocado.

Recientemente, Leibovitz et al ${ }^{26}$ estudiaron los signos y síntomas de OMA y su relación con la etiología bacteriana documentada por timpanocentesis. Los resultados del estudio confirmaron que no hay una diferencia significativa entre la clínica de una otitis producida por Haemophilus influenzae y una otitis por Streptococcus pneumoniae, demostrando, sin embargo, que la clínica de una otitis bacteriana es significativamente más severa que la de una otitis viral.

Otras técnicas diagnósticas, como la disminución de los niveles de factor de necrosis tumoral alfa como indicador de erradicación bacteriana y la medición de interleukina 8 como indicador de la presencia de $H$. influenzae ${ }^{31,32,33}$, están en investigación.

\section{Tratamiento}

En 1999 el Centro de Control de Enfermedades (CDC) de Estados Unidos convocó a un panel de expertos para formar el "Grupo de Trabajo para el Tratamiento de Streptococcus pneumoniae Resistente a Antibióticos”. Este, emitió recomendaciones para el tratamiento de otitis media en Estados Unidos, y estableció que la amoxicilina en una dosis de 45 $\mathrm{mg} / \mathrm{kg} /$ día, es la droga de primera elección en el manejo de otitis media aguda sin complicaciones, incluyendo dentro de este grupo a pacientes sin factores de riesgo de tener un Streptococcus pneumoniae resistente a penicilina. En aquellos pacientes considerados con alto riesgo de presentar una infección por un Streptococcus pneumoniae no susceptible a penicilina o en los catalogados como falla terapéutica, la recomendación fue la de iniciar tratamiento con alguna de las siguientes opciones: a-) amoxicilina en dosis de $90 \mathrm{mg} / \mathrm{kg}$ /día; b-) amoxicilina-ácido clavulánico (en dosis de 90/6.4 $\mathrm{mg} / \mathrm{kg} /$ día, respectivamente) o c-) cefuroxime axetil. En los que consulten por una falla terapéutica a estos medicamentos de segunda línea, la recomendación es utilizar tres dosis de ceftriaxona intramuscular ( $50 \mathrm{mg} / \mathrm{kg} /$ día, por 3 días $)$ o clin- damicina, y la realización de una timpanocentesis con fines terapéuticos y diagnósticos ${ }^{25}$.

Recientemente, la Academia Americana de Pediatría (AAP) emitió recomendaciones terapéuticas similares a las del grupo de trabajo del CDC. Estas indican que, en pacientes con una edad mayor a los 2 años, sin factores de riesgo (por ejemplo inmunosuprimidos) y con una otitis media leve, debe considerarse la posibilidad de un manejo sintomático, sin terapia antimicrobiana, utilizando medicamentos únicamente para la otalgia y la fiebre, más una nueva valoración a las 48-72 horas. Si esta conducta de "observación" se sigue en determinado paciente, el tratamiento antimicrobiano debe iniciarse en caso de que empeore su sintomatología durante el periodo de observación o si a las 72 horas no hay evidencia de mejoría clínica. En pacientes con edades entre los 6 meses y 2 años de edad, la AAP sugiere la conducta de "observación" únicamente para aquellos en los que existan dudas sobre el diagnóstico de otitis media, con una revaloración a las 24 horas y tratamiento con antibióticos ante la certeza de que tengan una otitis media ${ }^{34}$.

Con base en los datos de la microbiología obtenidos en niños costarricenses ${ }^{22,23}$, nuestra recomendación sería la de utilizar amoxicilina como antibiótico de primera elección, en una dosis de $50 \mathrm{mg} / \mathrm{kg} /$ día, por 10 días, en pacientes con otitis media no complicada y con bajo riesgo de tener un Streptococcus pneumoniae resistente a la penicilina. Para casos de alto riesgo de tener un Streptococcus pneumoniae resistente a la penicilina, pacientes con OMR o con OMF, nuestra recomendación es iniciar con alguna de las siguientes opciones: a-) amoxicilina en dosis de $90 \mathrm{mg} / \mathrm{kg} / \mathrm{día}$, por 10 días; b) amoxicilina con ácido clavulánico a 90mg/6.4 mg/kg/día, por 10 días, o alguno de los nuevos macrólidos (azitromicina o claritromicina) en dosis convencionales. Si a pesar de estas recomendaciones no hay una mejoría clínica, debe considerarse la utilización de ceftriaxona a $50 \mathrm{mg} / \mathrm{kg} /$ día, por 3 días. (Cuadro 1) ${ }^{24}$. La utilización de ceftriaxona debe reservarse como terapia de tercera línea para los pacientes que no han respondido a alguna de las opciones recomendadas o para los que no toleren la vía oral; esto con el objetivo de disminuir la posibilidad de selección de resistencia a cefalosporinas de tercera generación, que son antibióticos de gran valor para el tratamiento de pacientes con patologías más complejas.

Dentro de los agentes que se consideran como antibióticos de tercera línea están las fluoroquinolonas. Este grupo de antibióticos no está todavía aprobado para la población pediátrica, debido a la posibilidad de que estos puedan producir daño en los núcleos de crecimiento óseo. A pesar de lo anterior, a la fecha no se ha descrito la presencia de ningún problema de artropatía en niños que han utilizado quinolonas en múltiples estudios retrospectivos y prospectivos ${ }^{35,36}$. Estos datos hacen muy probable que, durante el 2004, tales antibióticos sean aprobados por la Administración de Drogas y Alimentos de los Estados Unidos (FDA) para su uso en niños, y que dentro de las indicaciones éste el tratamiento de OMR y 


\begin{tabular}{|c|c|c|}
\hline \multicolumn{3}{|c|}{$\begin{array}{c}\text { Cuadro 1: Recomendaciones para el tratamiento } \\
\text { de la otitis media en Costa Rica }\end{array}$} \\
\hline Enfermedad & Día 1 & $\begin{array}{c}\text { Alternativa en caso de } \\
\text { falla clínica a } \\
\text { selección en día } 1\end{array}$ \\
\hline $\begin{array}{l}\text { Otitis media } \\
\text { aguda }\end{array}$ & $\begin{array}{l}\text { Amoxicilina } \\
50 \text { mg/Kg/día } \\
\text { x } 10 \text { días }\end{array}$ & $\begin{array}{c}\text { Alternativas (una): } \\
\text { Amoxicilina dosis altas } \\
\text { (90 mg/Kg/día x } 10 \text { días): } \\
\text { amoxicilina-ácido } \\
\text { clavulánico } \\
\text { (90mg/6.4mg x Kg x día } \\
\text { x } 10 \text { días), macrólido, } \\
\text { ceftriaxona o clindamicina. } \\
\text { Considerar una timpanocentesis }\end{array}$ \\
\hline $\begin{array}{l}\text { Otitis media } \\
\text { recurrente }\end{array}$ & $\begin{array}{c}\text { Alternativas (una): } \\
\text { Amoxicilina en dosis } \\
\text { altas ( } 90 \mathrm{mg} / \mathrm{Kg} / \mathrm{día} \\
\text { x } 10 \text { días), } \\
\text { Amoxicilina- ácido } \\
\text { clavulánico } \\
\text { (90mg/6.4mg x Kg } \\
\text { x día x } 10 \text { días) } \\
\text { o un macrólido. }\end{array}$ & $\begin{array}{l}\text { Igual que } \\
\text { cuadro superior }\end{array}$ \\
\hline
\end{tabular}

a Se debe considerar una falla clínica si el paciente no tiene mejoría clínica 72 horas después de iniciado un antibiótico, o si hay una recaída antes del día 15 después de terminado el ciclo recomendado en el día 1.

OMF. Dos estudios recientes ${ }^{35,36}$ demuestran la excelente eficacia bacteriológica y clínica de uno de estos antibióticos.

\section{Prevención}

La prevención de la otitis debe ser un campo de interés, tanto para la salud pública como para médicos y padres de familia. Las estrategias de prevención se dividen en inmunoprofilácticas y no inmunoprofilácticas. Entre las no inmunoprofilácticas, encontramos que la educación a los padres es de gran importancia para la prevención de la otitis media, por lo que, el médico debe ser capaz de informar a los padres en los siguientes aspectos: 1) que la otitis media es una enfermedad de la infancia y que un niño, en promedio, puede tener de dos a tres episodios de otitis media por año durante los primeros tres años de vida; 2) que la predisposición familiar es un factor de riesgo para otitis recurrente y severa; 3) que la lactancia materna protege contra la otitis media; 4) que las guarderías aumentan la exposición a patógenos resistentes a antibióticos y 5) que la nicotina produce irritación del tracto respiratorio de todas las personas en contacto directo o indirecto con esta.

\section{Profilaxis antibiótica}

En cuanto a la profilaxis con antibióticos, un metaanálisis documentó que la administración de sulfisoxazol, trimetoprim-sulfametoxazol o amoxicilina, disminuye un promedio de 0.11 episodios, por niño, por mes, o alrededor de 1 episodio de otitis media aguda por año. A este pequeño beneficio se le superpone la desventaja de alergias, toxicidad y de promover la resistencia a antibióticos ${ }^{37}$. Por esta razón, en la actualidad la profilaxis antimicrobiana no se recomienda de rutina.

La efectividad de la vacuna del virus influenza en la prevención de otitis media ha sido estudiada en Finlandia y Estados Unidos ${ }^{38}$, se ha demostrado una reducción del $35 \%$ en la incidencia de episodios de otitis media, en niños vacunados contra influenza durante la temporada de este virus. Otro estudio indicó una reducción de un $40 \%$ en la incidencia de otitis media en pacientes tratados con oseltamivir, comparado con placebo, durante la temporada del virus mencionado ${ }^{39}$. En contraste, una investigación reciente sugiere que la vacuna de influenza no mostró ningún impacto sobre la incidencia de la otitis media ${ }^{40}$. Este último estudio tuvo el gran problema metodológico de que durante el tiempo de seguimiento de los participantes no hubo una estación con números importantes de infecciones por influenza, lo que produjo una dificultad para interpretar los resultados. Debido a lo anterior, la vacuna anual de influenza fue recomendada por la Academia Americana de Pediatría, para niños con edades entre los 6 meses y los 2 años ${ }^{41}$.

\section{Vacuna conjugada de Streptococcus pneumoniae}

La vacuna no conjugada de polisacáridos contra $S$. pneumoniae (Pneumovax $($ )), no es inmunogénica en niños menores de dos años. Recientemente fue aprobada una vacuna conjugada contra S. pneumoniae (Prevenar®), que contiene los siete serotipos más frecuentes (responsables del 83\% de las infecciones pneumocóccicas invasivas) ${ }^{42}$. Esta vacuna se puede aplicar a pacientes a partir de los dos meses de edad.

Dos estudios importantes han demostrado la importancia de esta vacuna en la prevención de episodios de otitis en niños menores de dos años. En ambos se evidenció una reducción del 6 al 9\% de todos los casos de OMA, una reducción del $33 \%$ en el número global de casos de OMA por cualquier $S$. pneumoniae y del $57 \%$ de los casos de OMA producidos por los serotipos incluidos en la vacuna. Además, se refirió una disminución del $20 \%$ en el número de episodios de otitis recurrentes, y del $22 \%$ en la colocación de tubos timpánicos ${ }^{3,43}$.

La profilaxis quirúrgica con adenoidectomía y tubos timpánicos está recomendada solo en casos selectos en los que, a pesar del manejo mencionado, el paciente persiste con episodios recurrentes de otitis media o problemas de audición o del habla. 


\section{Conclusión}

Con base en la bacteriología de nuestro país y las recomendaciones señaladas en estas observaciones, la amoxicilina en dosis de 50mg/Kg/día, por 10 días, debe ser el antibiótico de primera elección en el tratamiento de otitis media aguda no complicada. El manejo de pacientes con OMR, OMF y que muestren factores de riesgo para presentar una infección por un $S$. pneumoniae resistente a la penicilina, debe incluir la amoxicilina a dosis más altas o los antibióticos denominados de segunda línea.

Como medida preventiva, la vacuna contra la influenza y la vacuna conjugada de $S$. pneumoniae han tenido un impacto positivo en los países donde se han implementado, por lo que su uso debe considerarse también en el nuestro.

\section{A bstract}

Otitis media is one of the most common causes of consultation among the pediatric population. The pathogens most frequently isolated from middle ear fluid are Streptococcus pneumoniae, Haemophilus influenzae, Moraxella catarrhalis and Streptococcus pyogenes. Antimicrobial resistance patterns vary by region and their knowledge is considered important in order to achieve proper antimicrobial selection. In Costa Rica the microbiology of patients with acute, recurrent and treatment failure otitis media has been recently analyzed. The results of these studies indicate that amoxicillin at 50 $\mathrm{mg} / \mathrm{kg} /$ day for 10 days should be considered first line of therapy for patients with acute otitis media. In patients with recurrent otitis media, therapeutic failures or in patients at risk of having a penicillin non-susceptible Streptococcus pneumoniae, one of the second line agents should be considered.

\section{Referencias}

1. Klein JO, Bluestone CD. Acute otitis media. Pediatr Infect Dis J1982;1: 66-73

2. Robert JE, Sanyal MA, Burchinal MR, Collier AM, Ramey CT, Henderson FW. Otitis media in early childhood and its relationship to later verbal and academic performance. Pediatrics 1986;78: 423-30

3. Eskola J, Kilpi T, Palmu A, Jokinen J, Haapakoski J, Herva E, et al. Efficacy of a pneumococcal conjugate vaccine against acute otitis media. N Engl J Med, 2001;344: 403-409

4. Berman, S. Otitis media in children. N Engl J Med. 1995;332: 1560-5.

5. Freid VM, Makue DM, Rooks RN. Ambulatory health care visit by children: principal diagnosis and place of visit. Vital and health statistics. Series 13, No 137. Washington, DC.: Government Printing Office, 1998. (DHHS publication No.(PHS)98-1798)

6. Gates GA. Cost-effectiveness considerations in otitis media treatment. Otolaryngol Head Neck Surg. 1996;114: 525-30
7. Bondy J, Berman S, Glazner I, Lezotte D. Direct expenditures related to otitis media diagnoses: extrapolations from a pediatric Medicaid cohort. Pediatrics 2000;105: 1323.

8. Bluestone C. Pathogenesis of otitis media: role of Eustachian tube. Pediatr Infect Dis J1996;15: 281-291.

9. Ramilo O. Role of respiratory viruses in acute otitis media: implications for management Pediatr Infect Dis J1999; 18: 1125-1129.

10. Chonmautree T, Heikkinen T. Role of viruses in middle ear disease. Ann NYAcad Sci 1997;830: 143-157.

11. Sato K, Quartey MK, Liebeler CL, Le CT, Giebink GS. Role of autolysin and pneumolysin in middle ear inflammation caused by a type 3 Streptococcus pneumoniae strain in the chinchilla otitis media model. Infect Immun 1996;64: 1140-1145

12. Sato K, Liebeler C.L, Quartey M, Le, C.T, Giebink S. Middle ear fluid cytokine and inflammatory cell kinetics in the chinchilla otitis media model. Infect. Immun.1999;67: 1943-1946

13. Wang B, Cleary P, Xu H, Li JD. Up-Regulation of Interleukin 8 by novel small cytoplasmic molecules of non-typeable Haemophilus influenzae via p38 and extracellular signal-regulated kinase pathways. Infect Immun 2003;71: 5523-5530

14. Teele DW, Klein JO, Rosner B.. Epidemiology of otitis media during the first seven years of life in children in Greater Boston. J Infect Dis 1989;160: 83-94

15. Bruce P. Lanphear, Robert S. Byrd, Peggy Auinger, and Caroline B. Hall. Increasing prevalence of recurrent otitis media among children in the United States. Pediatrics 1997; 99: e1-7.

16. Black SB, Capra AM, Klein JO, Lieu TA, Martin KE, Shinefield HR. The cost of otitis media in a managed care population. 39th International Conference on Antimicrobial Agents and Chemotherapy (9/26/1999 through 9/29/1999).

17. Alho O, Laara E, Oja $\mathrm{H}$. What is the natural history of recurrent acute otitis media in infancy. J Fam Prac 1996;43: 258-64

18. Daly KA, Brown JE, Lindgren BR, Meland MH, Le CT, Giebink GS. Epidemiology of otitis media onset by six months of age. Pediatrics 1999;103: 1158-66

19. Uhari M, Mantysaari K, Niemela M. Ameta-analytic review of the risk factors for acute otitis media. Clin Infect Dis 1996;22: 1079-83

20. Casselbrandt ML, Mandel EM, Fall PA, Rockette HE, Kurs-Lasky M, Bluestone $\mathrm{CD}$ et al. The heritability of otitis media: a twin and triplet study. JAMA1999;282: 2125-2130.

21. Jacobs M, Dagan R, Appelbaum PC, Burch D. Prevalence of antimicrobial-resistant pathogens in middle ear fluid: multinational study of 917 children with acute otitis media. Antimicrob Agents Chemother 1998;42: 589-595

22. Arguedas A, Loaiza C, Pérez A, Vargas F, Herrera M, Rodriguez G, et al. Microbiology of acute otitis media in Costa Rican children. Pediatr Infect Dis J 1998;17: 680-9

23. Arguedas A, Dagan R, Soley C, Loaiza C, Knudsen K, Porat N, et al. Microbiology of otitis media in Costa Rican children, 1999 through 2001. Pediatr Infect Dis J 2003; 22: 1-0

24. Rosenblut A, Bacterial and viral etiology of acute otitis media in Chilean children. Pediatr Infect Dis J 2001;20: 501-507

25. Dowell S, Butler JC, Giebink G.S, Jacobs M.R, Jernigan D, Musher D.M,et al. Acute otitis media: management and surveillance in an era of pneumococcal resistance. A report from the Drug-resistant Streptococcus pneumoniae Therapeutic Working Group. Pediatr Infect Dis J 1999;18: 1-9

26. Leibovitz E, Satran R, Piglansky L, Raiz S, Press J, Leiberman Aet al. Can acute otitis media caused by Haemophilus influenzae be distinguished from that caused by Steptococcus pneumoniae. Pediatr infect Dis J 2003; 22: 509-514

27. Owen J. Otitis Media. N Engl J Med. 2002; 347: 1169-1174 
28. Dowell SF, Marcy S, Phillips WR, Gerber MA, Schwartz B. Otitis media principles of judicious use of antimicrobial agents. Pediatrics 1998;101: Suppl: 165-71

29. Chan LS, Takata GS, Shekelle P, Morton SC, Mason W, Marcy SM. Evidence assessment of management of acute otitis media. The role of antibiotics in treatment of uncomplicated acute otitis media. Pediatrics 2001;108: 239-47

30. Glasziou P, Mar C, Sanders S, Hayem M. Antibiotics for acute otitis media in children. Cochrane Database Syst Rev 2001;4

31. Barzilai A, Leibovitz E, Laver JH, Piglansky L, Raiz S, Abboud MR, et al. Dynamics of interleukin-1 production in middle ear fluid durinsg acute otitis media treated with antibiotics. Infection 1999;18: 173-6

32. Barzilai A, Leibovitz E, Laver JH, Piglansky L, Raiz S, Abboud MR, et al. Cytokine analysis of middle ear effusions during acute otitis media. Significant reduction in tumor necrosis factor alpha concentrations correlates with bacterial eradication. Pediatric Infect Dis J 1999;18: 301-3

33. Leibovitz E, Dagan R, Laver JH, Piglansky L, Raiz S, Abboud MR, et al. Interleukin- 8 in middle ear fluid during acute otitis media: correlation with etiology and bacterial eradication. Arch Dis Child 2000; 82: $165-8$

34. American Academy of Pediatrics. Diagnosis and management of acute otitis media. http://www.aap.org/advocacy/releases/maraomrelease.htm

35. Arguedas A, Sher L, Lopez E, Sáez-Llorens X, Hamed K, Skuba K, et al. Open label multicenter study of gatifloxacin treatment of recurrent otitis media and acute otitis media treatment failure. Pediatr Infect Dis J. 2003; 22: 949-55

36. Leibovitz E, Piglansky L, Raiz S, Greenberg D, Hamed KA, Ledeine $\mathrm{JM}$, et al. Bacteriologic and clinical efficacy of oral gatifloxacin for the treatment of recurrent/nonresponsive acute otitis media: an open label, non comparative, double tympanocentesis study. Pediatr Infect Dis J Nov 2003; 22: 943-949

37. Williams RL, Chalmers TC, Stange KC, Chalmers FT, Bowlin SJ.. Use of antibiotics in preventing recurrent otitis media and in treating otitis media with effusion: a meta - analytic attempt to resolve the brouhaha. JAMA1993; 270: 1344-51
38. Clements DA, Langdon L, Bland C, Walter E. Avaccine decreases the incidence of otitis media in 6 to 30 month old children in day care. Arch Pediatr Adolesc Med 1995; 149: 1113-7

39. Winther B, et al. Oral oseltamivir reduces the risk of developing acute otitis media (AOM) following influenza infection in children. Washington, D.C.: American Society for Microbiology, 2000: 480.

40. Hoberman A, Greenberg DP, Paradice JL, Rockette HE, Lave JR, Kearney $\mathrm{DH}$, et al. Effectiveness of inactivated influenza vaccine in preventing acute otitis media in young children: Arandomized controlled trial. JAMA2003; 290: 1608-16

41. Zangwill KM, Belshe RB. Safety and efficacy of trivalent inactivated influenza vaccine in young children: a summary for the new era of routine vaccination. Pediatr Infect Dis J, 2004;23: 189-200

42. Black S, Shinefield H, Fireman B, Lewis E, Ray P, Hansen JR, et al. Efficacy, safety and immunogenicity of heptavalent pneumococcal conjugate vaccine in children. Pediatr Infect Dis J 2000; 19: 187-195

43. Fireman B, Black S, Shinefield H, Lee J, Lewis E, Ray P. Impact of the pneumococcal conjugate vaccine on otitis media. Pediatr Infect Dis J 2003; 22: 10-16

44. Gehanno P, Panajotopoulos A, Barry B, Nguyen L, Levy D, Bingen E, et al. Microbiology of otitis media in the Paris, France area from 1987 to 1997. Pediatr Infect Dis J 2001; 20: 570-573.

45. Bluestone CD, Stephenson JS, Martin LM. Ten year review of otitis media pathogens. Pediatr Infect Dis J 1992;11: S7-11

46. Turner D, Leibovitz E, Aran A, Piglansky L, Raiz S, Leiberman A, et al. Acute otitis media in infants younger than two months of age: microbiology, clinical presentation and therapeutic approach. Pediatr Infect Dis J 21: 669-674

47. Kilpi T, Herva E, Kaijalainen T, Syrjanen R, Takala A. Bacteriology of acute otitis media in a cohort of Finnish children followed for the first two years of life. Pediatr Infect Dis J.2001; 20: 654-662. 